\title{
CONVEX FOLIATIONS OF DEGREE 4 ON THE COMPLEX PROJECTIVE PLANE
}

\author{
by
}

\author{
Samir BEDROUNI \& David MARÍN
}

\begin{abstract}
We show that up to automorphism of $\mathbb{P}_{\mathbb{C}}^{2}$ there are 5 homogeneous convex foliations of degree four on $\mathbb{P}_{\mathbb{C}}^{2}$. Using this result, we give a partial answer to a question posed in 2013 by D. MARín and J. PEREIRA about the classification of reduced convex foliations on $\mathbb{P}_{\mathbb{C}}^{2}$.

2010 Mathematics Subject Classification. - 37F75, 32S65, 32M25.
\end{abstract}

\section{Introduction}

The set $\mathbf{F}(d)$ of foliations of degree $d$ on $\mathbb{P}_{\mathbb{C}}^{2}$ can be identified with a ZARISKI open subset of the projective space $\mathbb{P}_{\mathbb{C}}^{(d+2)^{2}-2}$. The group of automorphisms of $\mathbb{P}_{\mathbb{C}}^{2}$ acts on $\mathbf{F}(d)$. The orbit of an element $\mathcal{F} \in \mathbf{F}(d)$ under the action of $\operatorname{Aut}\left(\mathbb{P}_{\mathbb{C}}^{2}\right)=\operatorname{PGL}_{3}(\mathbb{C})$ will be denoted by $O(\mathcal{F})$. Following [9] we will say that a foliation in $\mathbf{F}(d)$ is convex if its leaves other than straight lines have no inflection points. The subset $\mathbf{F C}(d)$ of $\mathbf{F}(d)$ consisting of all convex foliations is ZARISKI closed in $\mathbf{F}(d)$.

By [2, Proposition 2, page 23] every foliation of degree 0 or 1 is convex, i.e. $\mathbf{F C}(0)=\mathbf{F}(0)$ and $\mathbf{F C}(1)=\mathbf{F}(0)$. For $d \geq 2, \mathbf{F C}(d)$ is a proper closed subset of $\mathbf{F}(d)$ and it contains the FERMAT foliation $\mathcal{F}_{0}^{d}$ of degree $d$, defined in the affine chart $(x, y)$ by the 1-form (see [9, page 179])

$$
\bar{\omega}_{0}^{d}=\left(x^{d}-x\right) \mathrm{d} y-\left(y^{d}-y\right) \mathrm{d} x .
$$

The closure inside $\mathbf{F}(d)$ of the orbit of $\mathcal{F}_{0}^{d}$ contains the foliations $\mathcal{H}_{0}^{d}$, resp. $\mathcal{H}_{1}^{d}$, resp. $\mathcal{F}_{1}^{d}$ (necessarily convex) defined by the 1 -forms (see [3, Example 6.5] and [4, page 75])

$$
\omega_{0}^{d}=(d-1) y^{d} \mathrm{~d} x+x\left(x^{d-1}-d y^{d-1}\right) \mathrm{d} y, \quad \text { resp. } \omega_{1}^{d}=y^{d} \mathrm{~d} x-x^{d} \mathrm{~d} y, \quad \text { resp. } \bar{\omega}_{1}^{d}=y^{d} \mathrm{~d} x+x^{d}(x \mathrm{~d} y-y \mathrm{~d} x) .
$$

In other words, we have the following inclusions

$$
O\left(\mathcal{H}_{0}^{d}\right) \cup O\left(\mathcal{H}_{1}^{d}\right) \cup O\left(\mathcal{F}_{0}^{d}\right) \cup O\left(\mathcal{F}_{1}^{d}\right) \subset \overline{O\left(\mathcal{F}_{0}^{d}\right)} \subset \mathbf{F C}(d) .
$$

Key words and phrases. - convex foliation, homogeneous foliation, singularity, inflection divisor.

D. Marín acknowledges financial support from the Spanish Ministry of Economy and Competitiveness, through grant MTM2015-66165-P and the "María de Maeztu" Programme for Units of Excellence in R\&D (MDM-2014-0445). 
The foliations $\mathcal{H}_{0}^{d}$ and $\mathcal{H}_{1}^{d}$ are homogeneous, i.e. they are invariant by homotheties; moreover, they are linearly conjugated for $d=2$, but not for $d \geq 3$, see [3]. The dimension of the orbit of $\mathcal{F}_{1}^{d}$ is 6 [4], which is the least possible dimension in any degree $d$ greater or equal to 2 ([6, Proposition 2.3]). Notice (see [4]) that this bound is also attained by the non convex foliation $\mathcal{F}_{2}^{d}$ defined by the 1 -form

$$
\bar{\omega}_{2}^{d}=x^{d} \mathrm{~d} x+y^{d}(x \mathrm{~d} y-y \mathrm{~d} x) .
$$

The classification of the elements of $\mathbf{F C}(2)$ has been established by C. FAVRE and J. PEREIRA [8, Proposition 7.4]: up to automorphism of $\mathbb{P}_{\mathbb{C}}^{2}$, the foliations $\mathcal{H}_{0}^{2}, \mathcal{F}_{0}^{2}$ and $\mathcal{F}_{1}^{2}$ are the only convex foliations of degree 2 on $\mathbb{P}_{\mathbb{C}}^{2}$. This classification implies that in degree 2 the inclusions $(0.1)$ are equalities:

$$
\mathbf{F C}(2)=\overline{O\left(\mathcal{F}_{0}^{2}\right)}=O\left(\mathcal{H}_{0}^{2}\right) \cup O\left(\mathcal{F}_{0}^{2}\right) \cup O\left(\mathcal{F}_{1}^{2}\right) .
$$

For dimensional reasons the orbits $O\left(\mathcal{F}_{1}^{d}\right)$ and $O\left(\mathcal{F}_{2}^{d}\right)$ are closed; combining equalities (0.2) with [6, Theorem 3], we see, in particular, that the only closed orbits in $\mathbf{F}(2)$ by the action of $\operatorname{Aut}\left(\mathbb{P}_{\mathbb{C}}^{2}\right)$ are those of $\mathcal{F}_{1}^{2}$ and $\mathcal{F}_{2}^{2}$.

Convex foliations of degree 3 has been classified by the first author in his thesis [4, Corollary C]: every foliation $\mathcal{F} \in \mathbf{F C}(3)$ is linearly conjugated to one of the four foliations $\mathcal{H}_{0}^{3}, \mathcal{H}_{1}^{3}, \mathcal{F}_{0}^{3}$ or $\mathcal{F}_{1}^{3}$. This implies that the inclusions (0.1) for $d=3$ are also equalities:

$$
\mathbf{F C}(3)=\overline{O\left(\mathcal{F}_{0}^{3}\right)}=O\left(\mathcal{H}_{0}^{3}\right) \cup O\left(\mathcal{H}_{1}^{3}\right) \cup O\left(\mathcal{F}_{0}^{3}\right) \cup O\left(\mathcal{F}_{1}^{3}\right) .
$$

For $d \geq 4$, the classification of the elements of $\mathbf{F C}(d)$ modulo $\operatorname{Aut}\left(\mathbb{P}_{\mathbb{C}}^{2}\right)$ remains open and the topological structure of $\mathbf{F C}(d)$ is not yet well understood. In the sequel we will focus on the case $d=4$. Notice (see [9, page 181]) that the set $\mathbf{F C}(4)$ contains the foliation $\mathcal{F}_{H}^{4}$, called HESSE pencil of degree 4, defined by

$$
\omega_{H}^{4}=\left(2 x^{3}-y^{3}-1\right) y \mathrm{~d} x+\left(2 y^{3}-x^{3}-1\right) x \mathrm{~d} y ;
$$

furthermore $O\left(\mathcal{F}_{H}^{4}\right) \neq O\left(\mathcal{F}_{0}^{4}\right)$ and $\operatorname{dim} O\left(\mathcal{F}_{H}^{4}\right)=\operatorname{dim} O\left(\mathcal{F}_{0}^{4}\right)=8$. So that the inclusion $\overline{O\left(\mathcal{F}_{0}^{4}\right)} \subset \mathbf{F C}(4)$ is strict, in contrast to the previous cases of degrees 2 and 3.

In this paper we propose to classify, up to automorphism, the foliations of $\mathbf{F C}(4)$ which are homogeneous. More precisely, we establish the following theorem.

Theorem A. - Up to automorphism of $\mathbb{P}_{\mathbb{C}}^{2}$ there are five homogeneous convex foliations of degree four $\mathcal{H}_{1}, \ldots, \mathcal{H}_{5}$ on the complex projective plane. They are respectively described in affine chart by the following 1-forms

1. $\omega_{1}=y^{4} \mathrm{~d} x-x^{4} \mathrm{~d} y$

2. $\omega_{2}=y^{3}(2 x-y) \mathrm{d} x+x^{3}(x-2 y) \mathrm{d} y$;

3. $\omega_{3}=y^{2}\left(6 x^{2}+4 x y+y^{2}\right) \mathrm{d} x-x^{3}(x+4 y) \mathrm{d} y$;

4. $\omega_{4}=y^{3}(4 x+y) \mathrm{d} x+x^{3}(x+4 y) \mathrm{d} y$;

5. $\omega_{5}=y^{2}\left(6 x^{2}+4 x y+y^{2}\right) \mathrm{d} x+3 x^{4} \mathrm{~d} y$.

By [10] we know that every foliation of degree $d \geq 1$ on $\mathbb{P}_{\mathbb{C}}^{2}$ can not have more than $3 d$ (distinct) invariant lines. If this bound is reached for $\mathcal{F} \in \mathbf{F}(d)$, then $\mathcal{F}$ necessarily belongs to $\mathbf{F C}(d)$; in this case we say that $\mathcal{F}$ is reduced convex. To our knowledge the only reduced convex foliations known in the literature are those presented in [9, Table 1.1]: the FERMAT foliation $\mathcal{F}_{0}^{d}$ in any degree, the HESSE pencil $\mathcal{F}_{H}^{4}$ and the foliations given by the 1 -forms

$$
\begin{gathered}
\left(y^{2}-1\right)\left(y^{2}-(\sqrt{5}-2)^{2}\right)(y+\sqrt{5} x) \mathrm{d} x-\left(x^{2}-1\right)\left(x^{2}-(\sqrt{5}-2)^{2}\right)(x+\sqrt{5} y) \mathrm{d} y, \\
\left(y^{3}-1\right)\left(y^{3}+7 x^{3}+1\right) y \mathrm{~d} x-\left(x^{3}-1\right)\left(x^{3}+7 y^{3}+1\right) x \mathrm{~d} y,
\end{gathered}
$$


which have degrees 5 and 7 respectively. D. MARín and J. PereIRA [9, Problem 9.1] asked the following question: are there other reduced convex foliations? The answer in degree 2, resp. 3, to this question is negative, by [8, Proposition 7.4], resp. [3, Corollary 6.9]. Theorem A allows us to show that the answer to [9, Problem 9.1] in degree 4 is also negative.

Theorem B. - Up to automorphism of $\mathbb{P}_{\mathbb{C}}^{2}$, the FERMAT foliation $\mathcal{F}_{0}^{4}$ and the HESSE pencil $\mathcal{F}_{H}^{4}$ are the only reduced convex foliations of degree four on $\mathbb{P}_{\mathbb{C}}^{2}$.

\section{Preliminaries}

1.1. Singularities and inflection divisor of a foliation on the projective plane. - A degree $d$ holomorphic foliation $\mathcal{F}$ on $\mathbb{P}_{\mathbb{C}}^{2}$ is defined in homogeneous coordinates $[x: y: z]$ by a 1 -form

$$
\omega=a(x, y, z) \mathrm{d} x+b(x, y, z) \mathrm{d} y+c(x, y, z) \mathrm{d} z,
$$

where $a, b$ and $c$ are homogeneous polynomials of degree $d+1$ without common factor and satisfying the EULER condition $i_{\mathrm{R}} \omega=0$, where $\mathrm{R}=x \frac{\partial}{\partial x}+y \frac{\partial}{\partial y}+z \frac{\partial}{\partial z}$ denotes the radial vector field and $i_{\mathrm{R}}$ is the interior product by $\mathrm{R}$. The singular locus $\operatorname{Sing} \mathcal{F}$ of $\mathcal{F}$ is the projectivization of the singular locus of $\omega$

$$
\text { Sing } \omega=\left\{(x, y, z) \in \mathbb{C}^{3} \mid a(x, y, z)=b(x, y, z)=c(x, y, z)=0\right\} .
$$

Let us recall some local notions attached to the pair $(\mathcal{F}, s)$, where $s \in \operatorname{Sing} \mathcal{F}$. The germ of $\mathcal{F}$ at $s$ is defined, up to multiplication by a unity in the local ring $O_{s}$ at $s$, by a vector field $\mathrm{X}=A(\mathrm{u}, \mathrm{v}) \frac{\partial}{\partial \mathrm{u}}+B(\mathrm{u}, \mathrm{v}) \frac{\partial}{\partial \mathrm{v}}$. The vanishing $\operatorname{order} v(\mathcal{F}, s)$ of $\mathcal{F}$ at $s$ is given by

$$
\boldsymbol{v}(\mathcal{F}, s)=\min \{v(A, s), v(B, s)\},
$$

where $\mathrm{v}(g, s)$ denotes the vanishing order of the function $g$ at $s$. The tangency order of $\mathcal{F}$ with a generic line passing through $s$ is the integer

$$
\tau(\mathcal{F}, s)=\min \left\{k \geq v(\mathcal{F}, s): \operatorname{det}\left(J_{s}^{k} \mathbf{X}, \mathbf{R}_{s}\right) \neq 0\right\},
$$

where $J_{s}^{k} \mathrm{X}$ denotes the $k$-jet of $\mathrm{X}$ at $s$ and $\mathrm{R}_{s}$ is the radial vector field centered at $s$. The MiLnOR number of $\mathcal{F}$ at $s$ is the integer

$$
\mu(\mathcal{F}, s)=\operatorname{dim}_{\mathbb{C}} O_{s} /\langle A, B\rangle,
$$

where $\langle A, B\rangle$ denotes the ideal of $O_{s}$ generated by $A$ and $B$.

The singularity $s$ is called radial of order $n-1$ if $v(\mathcal{F}, s)=1$ and $\tau(\mathcal{F}, s)=n$.

The singularity $s$ is called non-degenerate if $\mu(\mathcal{F}, s)=1$, or equivalently if the linear part $J_{s}^{1} \mathrm{X}$ of X possesses two non-zero eigenvalues $\lambda, \mu$. In this case, the quantity $\operatorname{BB}(\mathcal{F}, s)=\frac{\lambda}{\mu}+\frac{\mu}{\lambda}+2$ is called the BAUM-BotT invariant of $\mathcal{F}$ at $s$ (see [1]). By [5] there is at least a germ of curve $\mathcal{C}$ at $s$ which is invariant by $\mathcal{F}$. Up to local diffeomorphism we can assume that $s=(0,0) \mathrm{T}_{s} \mathcal{C}=\{\mathrm{v}=0\}$ and $J_{S}^{1} \mathrm{X}=\lambda \mathrm{u} \frac{\partial}{\partial \mathrm{u}}+(\varepsilon \mathrm{u}+\mu \mathrm{v}) \frac{\partial}{\partial \mathrm{v}}$, where we can take $\varepsilon=0$ if $\lambda \neq \mu$. The quantity $\operatorname{CS}(\mathcal{F}, \mathcal{C}, s)=\frac{\lambda}{\mu}$ is called the CAMACHO-SAD index of $\mathcal{F}$ at $s$ along $C$.

Let us also recall the notion of inflection divisor of $\mathcal{F}$. Let $\mathrm{Z}=E \frac{\partial}{\partial x}+F \frac{\partial}{\partial y}+G \frac{\partial}{\partial z}$ be a homogeneous vector field of degree $d$ on $\mathbb{C}^{3}$ non collinear to the radial vector field describing $\mathcal{F}$, i.e. such that $\omega=i_{\mathrm{R}} i_{Z} \mathrm{~d} x \wedge \mathrm{d} y \wedge \mathrm{d} z$. The inflection divisor of $\mathcal{F}$, denoted by $\mathrm{I}_{\mathcal{F}}$, is the divisor of $\mathbb{P}_{\mathbb{C}}^{2}$ defined by the homogeneous equation

$$
\left|\begin{array}{lll}
x & E & \mathrm{Z}(E) \\
y & F & \mathrm{Z}(F) \\
z & G & \mathrm{Z}(G)
\end{array}\right|=0 .
$$


This divisor has been studied in [10] in a more general context. In particular, the following properties has been proved.

1. On $\mathbb{P}_{\mathbb{C}}^{2} \backslash \operatorname{Sing} \mathcal{F}, \mathrm{I}_{\mathcal{F}}$ coincides with the curve described by the inflection points of the leaves of $\mathcal{F}$;

2. If $\mathcal{C}$ is an irreducible algebraic curve invariant by $\mathcal{F}$ then $\mathcal{C} \subset \mathrm{I}_{\mathcal{F}}$ if and only if $\mathcal{C}$ is an invariant line;

3. $\mathrm{I}_{\mathcal{F}}$ can be decomposed into $\mathrm{I}_{\mathcal{F}}=\mathrm{I}_{\mathcal{F}}^{\mathrm{inv}}+\mathrm{I}_{\mathcal{F}}^{\mathrm{tr}}$, where the support of $\mathrm{I}_{\mathcal{F}}^{\mathrm{inv}}$ consists in the set of invariant lines of $\mathcal{F}$ and the support of $\mathrm{I}_{\mathcal{F}}^{\mathrm{tr}}$ is the closure of the isolated inflection points along the leaves of $\mathcal{F}$;

4. The degree of the divisor $\mathrm{I}_{\mathcal{F}}$ is $3 d$.

The foliation $\mathcal{F}$ will be called convex if its inflection divisor $\mathrm{I}_{\mathcal{F}}$ is totally invariant by $\mathcal{F}$, i.e. if $\mathrm{I}_{\mathcal{F}}$ is a product of invariant lines.

1.2. Geometry of homogeneous foliations. - A foliation of degree $d$ on $\mathbb{P}_{\mathbb{C}}^{2}$ is said to be homogeneous if there is an affine chart $(x, y)$ of $\mathbb{P}_{\mathbb{C}}^{2}$ in which it is invariant under the action of the group of homotheties $(x, y) \longmapsto \lambda(x, y), \lambda \in \mathbb{C}^{*}$. Such a foliation $\mathcal{H}$ is then defined by a 1 -form

$$
\omega=A(x, y) \mathrm{d} x+B(x, y) \mathrm{d} y,
$$

where $A$ and $B$ are homogeneous polynomials of degree $d$ without common factor. This 1-form writes in homogeneous coordinates as

$$
z A(x, y) \mathrm{d} x+z B(x, y) \mathrm{d} y-(x A(x, y)+y B(x, y)) \mathrm{d} z .
$$

Thus the foliation $\mathcal{H}$ has at most $d+2$ singularities whose origin $O$ of the affine chart $z=1$ is the only singular point of $\mathcal{H}$ which is not situated on the line at infinity $L_{\infty}=\{z=0\}$; moreover $v(\mathcal{H}, O)=d$.

In the sequel we will assume that $d$ is greater than or equal to 2 . In this case the point $O$ is the only singularity of $\mathcal{H}$ having vanishing order $d$.

We know from [3] that the inflection divisor of $\mathcal{H}$ is given by $z \mathrm{C}_{\mathcal{H}} \mathrm{D}_{\mathcal{H}}=0$, where $\mathrm{C}_{\mathcal{H}}=x A+y B \in \mathbb{C}[x, y]_{d+1}$ denotes the tangent cone of $\mathcal{H}$ at the origin $O$ and $\mathrm{D}_{\mathcal{H}}=\frac{\partial A}{\partial x} \frac{\partial B}{\partial y}-\frac{\partial A}{\partial y} \frac{\partial B}{\partial x} \in \mathbb{C}[x, y]_{2 d-2}$. From this we deduce that:

(i) the support of the divisor $\mathrm{I}_{\mathcal{H}}^{\text {inv }}$ consists of the lines of the tangent cone $\mathrm{C}_{\mathcal{H}}=0$ and the line at infinity $L_{\infty}$;

(ii) the divisor $\mathrm{I}_{\mathcal{H}}^{\mathrm{tr}}$ decomposes as $\mathrm{I}_{\mathcal{H}}^{\mathrm{tr}}=\prod_{i=1}^{n} T_{i}^{\rho_{i}-1}$ for some number $n \leq \operatorname{deg} \mathrm{D}_{\mathcal{H}}=2 d-2$ of lines $T_{i}$ passing through $O, \rho_{i}-1$ being the inflection order of the line $T_{i}$.

Proposition 1.1 ([3], Proposition 2.2). - With the previous notations, for any point $s \in \operatorname{Sing} \mathcal{H} \cap L_{\infty}$, we have 1. $v(\mathcal{H}, s)=1$;

2. the line joining the origin $O$ to the point $s$ is invariant by $\mathcal{H}$ and it appears with multiplicity $\tau(\mathcal{H}, s)-1$ in the divider $\mathrm{D}_{\mathcal{H}}=0$, i.e.

$$
\mathrm{D}_{\mathcal{H}}=\mathrm{I}_{\mathcal{H}}^{\mathrm{tr}} \prod_{s \in \operatorname{Sing} \mathcal{H} \cap L_{\infty}} L_{s}^{\tau(\mathcal{H}, s)-1} .
$$

Definition 1.2 ([3]). - Let $\mathcal{H}$ be a homogeneous foliation of degree $d$ on $\mathbb{P}_{\mathbb{C}}^{2}$ having a certain number $m \leq$ $d+1$ of radial singularities $s_{i}$ of order $\tau_{i}-1,2 \leq \tau_{i} \leq d$ for $i=1,2, \ldots, m$. The support of the divisor $\mathrm{I}_{\mathcal{H}}^{\mathrm{tr}}$ consists of a certain number $n \leq 2 d-2$ of transverse inflection lines $T_{j}$ of order $\rho_{j}-1,2 \leq \rho_{j} \leq d$ for $j=1,2, \ldots, n$. We define the type of the foliation $\mathcal{H}$ by

$$
\mathcal{T}_{\mathcal{H}}=\sum_{i=1}^{m} \mathrm{R}_{\tau_{i}-1}+\sum_{j=1}^{n} \mathrm{~T}_{\rho_{j-1}}=\sum_{k=1}^{d-1}\left(r_{k} \cdot \mathrm{R}_{k}+t_{k} \cdot \mathrm{T}_{k}\right) \in \mathbb{Z}\left[\mathrm{R}_{1}, \mathrm{R}_{2}, \ldots, \mathrm{R}_{d-1}, \mathrm{~T}_{1}, \mathrm{~T}_{2}, \ldots, \mathrm{T}_{d-1}\right] .
$$


Example 1.3. - Let us consider the homogeneous foliation $\mathcal{H}$ of degree 5 on $\mathbb{P}_{\mathbb{C}}^{2}$ defined by

$$
\omega=y^{5} \mathrm{~d} x+2 x^{3}\left(3 x^{2}-5 y^{2}\right) \mathrm{d} y .
$$

A straightforward computation leads to

$$
\mathrm{C}_{\mathcal{H}}=x y\left(6 x^{4}-10 x^{2} y^{2}+y^{4}\right) \quad \text { and } \quad \mathrm{D}_{\mathcal{H}}=150 x^{2} y^{4}(x-y)(x+y) .
$$

We see that the set of radial singularities of $\mathcal{H}$ consists of the two points $s_{1}=[0: 1: 0]$ and $s_{2}=[1: 0: 0]$; their orders of radiality are equal to 2 and 4 respectively. Moreover the support of the divisor $\mathrm{I}_{\mathcal{H}}^{\mathrm{tr}}$ is the union of the two lines $x-y=0$ and $x+y=0$; they are transverse inflection lines of order 1 . Therefore the foliation $\mathcal{H}$ is of type $\mathcal{T}_{\mathcal{H}}=1 \cdot \mathrm{R}_{2}+1 \cdot \mathrm{R}_{4}+2 \cdot \mathrm{T}_{1}$.

Following [3] to every homogeneous foliation $\mathcal{H}$ of degree $d$ on $\mathbb{P}_{\mathbb{C}}^{2}$ is associated a rational map from the RIEMANN sphere $\mathbb{P}_{\mathbb{C}}^{1}$ to itself of degree $d$ denoted by $\underline{\mathcal{G}}_{\mathcal{H}}$ and defined as follows: if $\mathcal{H}$ is described by $\omega=A(x, y) \mathrm{d} x+B(x, y) \mathrm{d} y$, with $A$ and $B$ being homogeneous polynomials of degree $d$ without common factor, the image of the point $[x: y] \in \mathbb{P}_{\mathbb{C}}^{1}$ by $\underline{G}_{\mathcal{H}}$ is the point $[-A(x, y): B(x, y)] \in \mathbb{P}_{\mathbb{C}}^{1}$. It is clear that this definition does not depend on the choice of the homogeneous 1-form $\omega$ describing the foliation $\mathcal{H}$. Notice that the map $\underline{\mathcal{G}}_{\mathcal{H}}$ has the following properties (see [3]):

1. the fixed points of $\underline{\mathcal{G}}_{\mathcal{H}}$ correspond to the tangent cone of $\mathcal{H}$ at the origin $O$ (i.e. $[a: b] \in \mathbb{P}_{\mathbb{C}}^{1}$ is fixed by $\underline{\mathcal{G}}_{\mathcal{H}}$ if and only if the line by $-a x=0$ is invariant by $\mathcal{H}$ );

2. the point $[a: b] \in \mathbb{P}_{\mathbb{C}}^{1}$ is a fixed critical point of $\underline{\mathcal{G}}_{\mathcal{H}}$ if and only if the point $[b: a: 0] \in L_{\infty}$ is a radial singularity of $\mathcal{H}$. The multiplicity of the critical point $[a: b]$ of $\underline{\mathcal{G}}_{\mathcal{H}}$ is exactly equal to the radiality order of the singularity at infinity;

3. the point $[a: b] \in \mathbb{P}_{\mathbb{C}}^{1}$ is a non-fixed critical point of $\underline{G}_{\mathcal{H}}$ if and only if the line $b y-a x=0$ is a transverse inflection line of $\mathcal{H}$. The multiplicity of the critical point $[a: b]$ of $\underline{\mathcal{G}}_{\mathcal{H}}$ is precisely equal to the inflection order of this line.

It follows, in particular, that a homogeneous foliation $\mathcal{H}$ on $\mathbb{P}_{\mathbb{C}}^{2}$ is convex if and only if its associated map $\underline{G}_{\mathcal{H}}$ has only fixed critical points; more precisely, a homogeneous foliation $\mathcal{H}$ of degree $d$ on $\mathbb{P}_{\mathbb{C}}^{2}$ is convex of type $\mathcal{T}_{\mathcal{H}}=\sum_{k=1}^{d-1} r_{k} \cdot \mathrm{R}_{k}$ if and only if the map $\underline{G}_{\mathcal{H}}$ possesses $r_{1}$, resp. $r_{2}, \ldots$, resp. $r_{d-1}$ fixed critical points of multiplicity 1 , resp. $2 \ldots$, resp. $d-1$, with $\sum_{k=1}^{d-1} k r_{k}=2 d-2$.

\section{Proof of Theorem A}

Before proving Theorem A, let us recall the next result which follows from Propositions 4.1 and 4.2 of [3]:

Proposition 2.1 ([3]). - Let $\mathcal{H}$ be a convex homogeneous foliation of degree $d \geq 3$ on $\mathbb{P}_{\mathbb{C}}^{2}$. Let $\vee$ be an integer between 1 and $d-2$. Then, $\mathcal{H}$ is of type

$$
\mathcal{T}_{\mathcal{H}}=2 \cdot \mathrm{R}_{d-1}, \quad \quad \quad \text { resp. } \mathcal{T}_{\mathcal{H}}=1 \cdot \mathrm{R}_{v}+1 \cdot \mathrm{R}_{d-v-1}+1 \cdot \mathrm{R}_{d-1},
$$

if and only if it is linearly conjugated to the foliation $\mathcal{H}_{1}^{d}$, resp. $\mathcal{H}_{3}^{d, v}$ given by

$$
\omega_{1}^{d}=y^{d} \mathrm{~d} x-x^{d} \mathrm{~d} y, \quad \text { resp. } \omega_{3}^{d, v}=\sum_{i=v+1}^{d}\left(\begin{array}{c}
d \\
i
\end{array}\right) x^{d-i} y^{i} \mathrm{~d} x-\sum_{i=0}^{v}\left(\begin{array}{c}
d \\
i
\end{array}\right) x^{d-i} y^{i} \mathrm{~d} y .
$$


Proof of Theorem A. - Let $\mathcal{H}$ be a convex homogeneous foliation of degree 4 on $\mathbb{P}_{\mathbb{C}}^{2}$, defined in the affine chart $(x, y)$, by the 1 -form

$$
\omega=A(x, y) \mathrm{d} x+B(x, y) \mathrm{d} y, \quad A, B \in \mathbb{C}[x, y]_{4}, \operatorname{gcd}(A, B)=1 .
$$

By [4, Remark 2.5] the foliation $\mathcal{H}$ can not have $4+1=5$ distinct radial singularities, in other words it can not be of type $4 \cdot R_{1}+1 \cdot R_{2}$. We are then in one of the following situations:

$$
\begin{array}{lll}
\mathcal{T}_{\mathcal{H}}=2 \cdot \mathrm{R}_{3} ; & \mathcal{T}_{\mathcal{H}}=1 \cdot \mathrm{R}_{1}+1 \cdot \mathrm{R}_{2}+1 \cdot \mathrm{R}_{3} ; & \mathcal{T}_{\mathcal{H}}=3 \cdot \mathrm{R}_{2} ; \\
\mathcal{T}_{\mathcal{H}}=2 \cdot \mathrm{R}_{1}+2 \cdot \mathrm{R}_{2} ; & & \mathcal{T}_{\mathcal{H}}=3 \cdot \mathrm{R}_{1}+1 \cdot \mathrm{R}_{3} .
\end{array}
$$

- If $\mathcal{T}_{\mathcal{H}}=2 \cdot \mathrm{R}_{3}$, resp. $\mathcal{T}_{\mathcal{H}}=1 \cdot \mathrm{R}_{1}+1 \cdot \mathrm{R}_{2}+1 \cdot \mathrm{R}_{3}$, then by [3, Propositions 4.1 , 4.2], the 1 -form $\omega$ is linearly conjugated to

$$
\omega_{1}^{4}=y^{4} \mathrm{~d} x-x^{4} \mathrm{~d} y=\omega_{1}
$$

resp. $\omega_{3}^{4,1}=\sum_{i=2}^{4}\left(\begin{array}{l}4 \\ i\end{array}\right) x^{4-i} y^{i} \mathrm{~d} x-\sum_{i=0}^{1}\left(\begin{array}{l}4 \\ i\end{array}\right) x^{4-i} y^{i} \mathrm{~d} y=y^{2}\left(6 x^{2}+4 x y+y^{2}\right) \mathrm{d} x-x^{3}(x+4 y) \mathrm{d} y=\omega_{3}$.

- Assume that $\mathcal{T}_{\mathcal{H}}=3 \cdot \mathrm{R}_{2}$. This means that the rational map $\underline{\mathcal{G}}_{\mathcal{H}}: \mathbb{P}_{\mathbb{C}}^{1} \rightarrow \mathbb{P}_{\mathbb{C}}^{1}, \underline{\mathcal{G}}_{\mathcal{H}}(z)=-\frac{A(1, z)}{B(1, z)}$, admits three different fixed critical points of multiplicity 2. By [7, page 79], $\underline{G}_{\mathcal{H}}$ is conjugated by a MöBIUS transformation to $z \mapsto-\frac{z^{3}(2-z)}{1-2 z}$. As a consequence, $\omega$ is linearly conjugated to

$$
\omega_{2}=y^{3}(2 x-y) \mathrm{d} x+x^{3}(x-2 y) \mathrm{d} y .
$$

- Assume that $\mathcal{T}_{\mathcal{H}}=2 \cdot \mathrm{R}_{1}+2 \cdot \mathrm{R}_{2}$. Then the rational map $\underline{G}_{\mathcal{H}}$ possesses four fixed critical points, two of them having multiplicity 1 and the other two having multiplicity 2 . This implies, by [7, page 79], that up to conjugation by a MöBıUs transformation, $\underline{\mathcal{G}}_{\mathcal{H}}$ writes as

$$
z \mapsto-\frac{z^{3}(2 z+3 c z-4 c-3)}{z+c},
$$

where $c=-3 / 8 \pm \sqrt{5} / 8$. Thus, up to linear conjugation

$$
\omega=y^{3}(2 y+3 c y-4 c x-3 x) \mathrm{d} x+x^{3}(y+c x) \mathrm{d} y, \quad c=-\frac{3}{8} \pm \frac{\sqrt{5}}{8} .
$$

In both cases $(c=-3 / 8+\sqrt{5} / 8$ or $c=-3 / 8-\sqrt{5} / 8)$, the 1 -form $\omega$ is linearly conjugated to

$$
\omega_{4}=y^{3}(4 x+y) \mathrm{d} x+x^{3}(x+4 y) \mathrm{d} y .
$$

Indeed,

$$
\omega_{4}=\frac{3 c+2}{2} \varphi^{*} \omega, \quad \text { where } \varphi=(2 x, 8 c y) .
$$

- Finally, consider the last situation: $\mathcal{T}_{\mathcal{H}}=3 \cdot \mathrm{R}_{1}+1 \cdot \mathrm{R}_{3}$. Up to linear conjugation we can assume that $\mathrm{D}_{\mathcal{H}}=c x^{3} y(y-x)(y-\alpha x)$ and $\mathrm{C}_{\mathcal{H}}(0,1)=\mathrm{C}_{\mathcal{H}}(1,0)=\mathrm{C}_{\mathcal{H}}(1,1)=\mathrm{C}_{\mathcal{H}}(1, \alpha)=0$, for some $c, \alpha \in \mathbb{C}^{*}, \alpha \neq$ 1 . The points $\infty=[1: 0],[0: 1],[1: 1],[1: \alpha] \in \mathbb{P}_{\mathbb{C}}^{1}$ are then fixed and critical for $\underline{\mathcal{G}}_{\mathscr{H}}$, having respective multiplicities 3, 1,1,1. By [3, Lemma 3.9], there exist constants $a_{0}, a_{2}, b \in \mathbb{C}^{*}, a_{1} \in \mathbb{C}$ such that $B(x, y)=b x^{4}, \quad A(x, y)=\left(a_{0} x^{2}+a_{1} x y+a_{2} y^{2}\right) y^{2}, \quad(z-1)^{2}$ divides $P(z), \quad(z-\alpha)^{2}$ divides $Q(z)$, 
where $P(z):=A(1, z)+B(1, z)$ and $Q(z):=A(1, z)+\alpha B(1, z)$. It follows that

$$
\left\{\begin{array} { l } 
{ P ( 1 ) = 0 } \\
{ P ^ { \prime } ( 1 ) = 0 } \\
{ Q ( \alpha ) = 0 } \\
{ Q ^ { \prime } ( \alpha ) = 0 }
\end{array} \Leftrightarrow \left\{\begin{array} { l } 
{ a _ { 0 } + a _ { 1 } + a _ { 2 } + b = 0 } \\
{ 2 a _ { 0 } + 3 a _ { 1 } + 4 a _ { 2 } = 0 } \\
{ a _ { 2 } \alpha ^ { 3 } + a _ { 1 } \alpha ^ { 2 } + a _ { 0 } \alpha + b = 0 } \\
{ 4 a _ { 2 } \alpha ^ { 2 } + 3 a _ { 1 } \alpha + 2 a _ { 0 } = 0 }
\end{array} \Leftrightarrow \left\{\begin{array}{l}
a_{0}=2 a_{2} \alpha \\
a_{1}=-\frac{4 a_{2}(\alpha+1)}{3} \\
b=-\frac{a_{2}(2 \alpha-1)}{3} \\
\alpha^{2}-\alpha+1=0
\end{array}\right.\right.\right.
$$

By replacing $\omega$ by $\frac{3}{a_{2}} \omega$, we can assume that

$$
\omega=y^{2}\left(6 \alpha x^{2}-4(\alpha+1) x y+3 y^{2}\right) \mathrm{d} x-(2 \alpha-1) x^{4} \mathrm{~d} y, \quad \alpha^{2}-\alpha+1=0 .
$$

The 1-form $\omega$ is linearly conjugated to

$$
\omega_{5}=y^{2}\left(6 x^{2}+4 x y+y^{2}\right) \mathrm{d} x+3 x^{4} \mathrm{~d} y .
$$

Indeed, the fact that $\alpha$ satisfies $\alpha^{2}-\alpha+1=0$ implies that

$$
\omega_{5}=\frac{1-\alpha}{(\alpha-2)^{3}} \varphi^{*} \omega, \quad \text { where } \varphi=((\alpha-2) x, y) .
$$

The foliations $\mathcal{H}_{i}, i=1, \ldots, 5$, are not linearly conjugated because, by construction, $\mathcal{T}_{\mathcal{H}_{j}} \neq \mathcal{T}_{\mathcal{H}_{i}}$ for each $j \neq i$. This ends the proof of the theorem.

A remarkable feature of the classification obtained is that all the singularities of the foliations $\mathcal{H}_{i}, i=1, \ldots, 5$, on the line at infinity are non-degenerated. In the following section we will need the values of the CAMACHO$\operatorname{SAD}$ indices $\operatorname{CS}\left(\mathcal{H}_{i}, L_{\infty}, s\right), s \in \operatorname{Sing} \mathcal{H}_{i} \cap L_{\infty}$. For this reason, we have computed, for each $i=1, \ldots, 5$, the following polynomial (called CAMACHO-SAD polynomial of the homogeneous foliation $\mathcal{H}_{i}$ )

$$
\mathrm{CS}_{\mathcal{H}_{i}}(\lambda)=\prod_{s \in \operatorname{Sing} \mathcal{H}_{i} \cap L_{\infty}}\left(\lambda-\operatorname{CS}\left(\mathcal{H}_{i}, L_{\infty}, s\right)\right) .
$$

The following table summarizes the types and the CAMACHO-SAD polynomials of the foliations $\mathcal{H}_{i}, i=$ $1, \ldots, 5$.

\begin{tabular}{|c|c|c|}
\hline$i$ & $\mathcal{T}_{\mathcal{H}_{i}}$ & $\mathrm{CS}_{\mathcal{H}_{i}}(\lambda)$ \\
\hline 1 & $2 \cdot \mathrm{R}_{3}$ & $(\lambda-1)^{2}\left(\lambda+\frac{1}{3}\right)^{3}$ \\
\hline 2 & $3 \cdot \mathrm{R}_{2}$ & $(\lambda-1)^{3}(\lambda+1)^{2}$ \\
\hline 3 & $1 \cdot \mathrm{R}_{1}+1 \cdot \mathrm{R}_{2}+1 \cdot \mathrm{R}_{3}$ & $(\lambda-1)^{3}\left(\lambda+\frac{13+2 \sqrt{13}}{13}\right)\left(\lambda+\frac{13-2 \sqrt{13}}{13}\right)$ \\
\hline 4 & $2 \cdot \mathrm{R}_{1}+2 \cdot \mathrm{R}_{2}$ & $(\lambda-1)^{4}(\lambda+3)$ \\
\hline 5 & $3 \cdot \mathrm{R}_{1}+1 \cdot \mathrm{R}_{3}$ & $(\lambda-1)^{4}(\lambda+3)$ \\
\hline
\end{tabular}

TABLE 1. Types and CAMACHO-SAD polynomials of the homogeneous foliations given by Theorem A. 


\section{Proof of Theorem B}

The proof of Theorem B is based on the classification of convex homogeneous foliations of degree four on $\mathbb{P}_{\mathbb{C}}^{2}$ given by Theorem $\mathrm{A}$ and on the three following results which hold in arbitrary degree.

First, notice that if $\mathcal{F}$ is a foliation of degree $d \geq 1$ on $\mathbb{P}_{\mathbb{C}}^{2}$ and if $s$ is a singular point of $\mathcal{F}$ then

$$
\sigma(\mathcal{F}, s) \leq \tau(\mathcal{F}, s)+1 \leq d+1
$$

where $\sigma(\mathcal{F}, s)$ denotes the number of (distinct) invariant lines of $\mathcal{F}$ passing through $s$.

The following lemma shows that the left-hand inequality above is an equality in the case where $\mathcal{F}$ is a reduced convex foliation.

Lemma 3.1. - Let $\mathcal{F}$ be a reduced convex foliation of degree $d \geq 1$ on $\mathbb{P}_{\mathbb{C}}^{2}$. Then, through each singular point $s$ of $\mathcal{F}$ pass exactly $\tau(\mathcal{F}, s)+1$ invariant lines of $\mathcal{F}$, i.e. $\sigma(\mathcal{F}, s)=\tau(\mathcal{F}, s)+1$.

Proof. - Let $s$ be a singular point of $\mathcal{F}$. Since the inflection divisor $\mathrm{I}_{\mathcal{F}}$ of $\mathcal{F}$ is totally invariant by $\mathcal{F}$ and it is reduced, we deduce that $\mu(\mathcal{F}, s)=1([3$, Lemma 6.8]) and the number $\sigma(\mathcal{F}, s)$ coincides with the vanishing order of $\mathrm{I}_{\mathcal{F}}$ at $s$. On the other hand, an elementary computation, using the equality $\mu(\mathcal{F}, s)=1$, shows that the vanishing order of $\mathrm{I}_{\mathcal{F}}$ at $s$ is equal to $\tau(\mathcal{F}, s)+1$. Hence the lemma holds.

The following result allows us to reduce the study of the convexity to the homogeneous framework:

Proposition 3.2. - Let $\mathcal{F}$ be a reduced convex foliation of degree $d \geq 1$ on $\mathbb{P}_{\mathbb{C}}^{2}$ and let $\ell$ be one of its $3 d$ invariant lines. There is a convex homogeneous foliation $\mathcal{H}$ of degree $d$ on $\mathbb{P}_{\mathbb{C}}^{2}$ satisfying the following properties:

(i) $\mathcal{H} \in \overline{O(\mathcal{F})}$;

(ii) $\ell$ is invariant by $\mathcal{H}$;

(iii) $\operatorname{Sing} \mathcal{H} \cap \ell=\operatorname{Sing} \mathcal{F} \cap \ell$;

(iv) $\forall s \in \operatorname{Sing} \mathcal{H} \cap \ell, \mu(\mathcal{H}, s)=1$;

(v) $\forall s \in \operatorname{Sing} \mathcal{H} \cap \ell, \tau(\mathcal{H}, s)=\tau(\mathcal{F}, s)$;

(vi) $\forall s \in \operatorname{Sing} \mathcal{H} \cap \ell, \operatorname{CS}(\mathcal{H}, \ell, s)=\operatorname{CS}(\mathcal{F}, \ell, s)$.

Proof. - We take a homogeneous coordinate system $[x: y: z] \in \mathbb{P}_{\mathbb{C}}^{2}$ such that $\ell=\{z=0\}$. Since $\ell$ is $\mathcal{F}$ invariant, $\mathcal{F}$ is defined in the affine chart $z=1$ by a 1 -form of the following type

$$
\omega=\sum_{i=0}^{d}\left(A_{i}(x, y) \mathrm{d} x+B_{i}(x, y) \mathrm{d} y\right),
$$

where $A_{i}, B_{i}$ are homogeneous polynomials of degree $i$. Using the fact that every reduced convex foliation on $\mathbb{P}_{\mathbb{C}}^{2}$ has only non-degenerate singularities ([3, Lemma 6.8]) and arguing as in the proof of [3, Proposition 6.4], we see that the 1 -form $\omega_{d}=A_{d}(x, y) \mathrm{d} x+B_{d}(x, y) \mathrm{d} y$ well defines a homogeneous foliation $\mathcal{H}$ of degree $d$ on $\mathbb{P}_{\mathbb{C}}^{2}$, and that this foliation satisfies the announced properties (i), (ii), (iii), (iv) and (vi). In particular, since $\mathcal{F}$ is convex by hypothesis, property (i) implies that $\mathcal{H}$ is also convex.

Let us show that $\mathcal{H}$ also satisfies property (v). Set $\Lambda:=\operatorname{Sing} \mathcal{H} \cap \ell=\operatorname{Sing} \mathcal{F} \cap \ell$; since $\mathcal{F}$ possesses $3 d$ invariant lines, we have $\sum_{s \in \Lambda}(\sigma(\mathcal{F}, s)-1)=3 d-1$. By Lemma 3.1, this is equivalent to $\sum_{s \in \Lambda} \tau(\mathcal{F}, s)=$ $3 d-1$. By [3, Proposition 2.2] the convexity of $\mathcal{H}$ implies that $\sum_{s \in \Lambda} \tau(\mathcal{H}, s)=2 d-2$. Moreover, the already proved property (iv) ensures that $\# \Lambda=d+1$. It follows that

$$
\sum_{s \in \Lambda}(\tau(\mathcal{F}, s)-1)=(3 d-1)-\# \Lambda=2 d-2=\sum_{s \in \Lambda}(\tau(\mathcal{H}, s)-1) .
$$


Thus, in order to see that $\mathcal{H}$ satisfies property (v), it is enough to prove that $\tau(\mathcal{F}, s) \leq \tau(\mathcal{H}, s)$ for each $s \in \Lambda$. Let us fix $s \in \Lambda$. Up to conjugating $\omega$ by a linear isomorphism of $\mathbb{C}^{2}=(z=1)$, we can assume that $s=[0: 1: 0]$. The foliations $\mathcal{F}$ and $\mathcal{H}$ are respectively defined in the affine chart $y=1$ by the 1 -forms

$$
\theta=\sum_{i=0}^{d} z^{d-i}\left[A_{i}(x, 1)(z \mathrm{~d} x-x \mathrm{~d} z)-B_{i}(x, 1) \mathrm{d} z\right] \quad \text { and } \quad \theta_{d}=A_{d}(x, 1)(z \mathrm{~d} x-x \mathrm{~d} z)-B_{d}(x, 1) \mathrm{d} z .
$$

As a consequence

$$
\tau(\mathcal{F}, s)=\min \left\{k \geq 1: J_{(0,0)}^{k}\left(\sum_{i=0}^{d} z^{d-i} B_{i}(x, 1)\right) \neq 0\right\} \leq \min \left\{k \geq 1: J_{0}^{k}\left(B_{d}(x, 1)\right) \neq 0\right\}=\tau(\mathcal{H}, s) .
$$

Remark 3.3. - If $\mathcal{F}$ is a foliation of degree $d$ on $\mathbb{P}_{\mathbb{C}}^{2}$ then (see [2])

$$
\sum_{s \in \operatorname{Sing} \mathcal{F}} \mu(\mathcal{F}, s)=d^{2}+d+1 \quad \text { and } \quad \sum_{s \in \operatorname{Sing} \mathcal{F}} \operatorname{BB}(\mathcal{F}, s)=(d+2)^{2} .
$$

Lemma 3.4. - Every foliation of degree $d \geq 1$ on $\mathbb{P}_{\mathbb{C}}^{2}$ possesses at least a non radial singularity.

This lemma follows from the formulas (3.1) and the obvious following remark: if a foliation $\mathcal{F}$ on $\mathbb{P}_{\mathbb{C}}^{2}$ admits a radial singularity $s$, then $\mu(\mathcal{F}, s)=1$ and $\mathrm{BB}(\mathcal{F}, s)=4$.

Proof of Theorem B. - Let $\mathcal{F}$ be a reduced convex foliation of degree 4 on $\mathbb{P}_{\mathbb{C}}^{2}$. Let us denote by $\Sigma$ the set of non radial singularities of $\mathcal{F}$. By Lemma $3.4, \Sigma$ is nonempty. Since by hypothesis $\mathcal{F}$ is reduced convex, all its singularities have MILNOR number 1 ([3, Lemma 6.8]). The set $\Sigma$ consists then of the singularities $s \in \operatorname{Sing} \mathcal{F}$ such that $\tau(\mathcal{F}, s)=1$. Let $m$ be a point of $\Sigma$; by Lemma 3.1, through the point $m$ pass exactly two $\mathcal{F}$-invariant lines $\ell_{m}^{(1)}$ and $\ell_{m}^{(2)}$.

On the other hand, for any line $\ell$ invariant by $\mathcal{F}$, Proposition 3.2 ensures the existence of a convex homogeneous foliation $\mathcal{H}_{\ell}$ of degree 4 on $\mathbb{P}_{\mathbb{C}}^{2}$ belonging to $\overline{O(\mathcal{F})}$ and such that the line $\ell$ is $\mathcal{H}_{\ell}$-invariant. Therefore $\mathcal{H}_{\ell}$, and in particular each $\mathcal{H}_{\ell_{m}^{(i)}}$, is linearly conjugated to one of the five homogeneous foliations given by Theorem A. Proposition 3.2 also ensures that

(a) $\operatorname{Sing} \mathcal{F} \cap \ell=\operatorname{Sing} \mathcal{H}_{\ell} \cap \ell$;

(b) $\forall s \in \operatorname{Sing} \mathcal{H}_{\ell} \cap \ell, \mu\left(\mathcal{H}_{\ell}, s\right)=1$;

(c) $\forall s \in \operatorname{Sing} \mathcal{H}_{\ell} \cap \ell, \tau\left(\mathcal{H}_{\ell}, s\right)=\tau(\mathcal{F}, s)$;

(d) $\forall s \in \operatorname{Sing} \mathcal{H}_{\ell} \cap \ell, \operatorname{CS}\left(\mathcal{H}_{\ell}, \ell, s\right)=\operatorname{CS}(\mathcal{F}, \ell, s)$.

Since $\operatorname{CS}\left(\mathcal{F}, \ell_{m}^{(1)}, m\right) \operatorname{CS}\left(\mathcal{F}, \ell_{m}^{(2)}, m\right)=1$, relation (d) implies that $\operatorname{CS}\left(\mathcal{H}_{\ell_{m}^{(1)}}, \ell_{m}^{(1)}, m\right) \operatorname{CS}\left(\mathcal{H}_{\ell_{m}^{(2)}}, \ell_{m}^{(2)}, m\right)=1$. This equality and Table 1 lead to

$$
\left\{\operatorname{CS}\left(\mathcal{H}_{\ell_{m}^{(1)}}, \ell_{m}^{(1)}, m\right), \operatorname{CS}\left(\mathcal{H}_{\ell_{m}^{(2)}}, \ell_{m}^{(2)}, m\right)\right\}=\left\{-3,-\frac{1}{3}\right\} \quad \text { or } \quad \operatorname{CS}\left(\mathcal{H}_{\ell_{m}^{(1)}}, \ell_{m}^{(1)}, m\right)=\operatorname{CS}\left(\mathcal{H}_{\ell_{m}^{(2)}}, \ell_{m}^{(2)}, m\right)=-1 .
$$

At first let us suppose that it is possible to choose $m \in \Sigma$ so that

$$
\left\{\mathrm{CS}\left(\mathcal{H}_{\ell_{m}^{(1)}}, \ell_{m}^{(1)}, m\right), \operatorname{CS}\left(\mathcal{H}_{\ell_{m}^{(2)}}, \ell_{m}^{(2)}, m\right)\right\}=\left\{-3,-\frac{1}{3}\right\} .
$$

By renumbering the $\ell_{m}^{(i)}$ we can assume that $\operatorname{CS}\left(\mathcal{H}_{\ell_{m}^{(1)}}, \ell_{m}^{(1)}, m\right)=-\frac{1}{3}$ and $\operatorname{CS}\left(\mathcal{H}_{\ell_{m}^{(2)}}, \ell_{m}^{(2)}, m\right)=-3$. Consulting Table 1, we see that

$$
\mathcal{T}_{\mathcal{H}_{\ell_{m}^{(1)}}}=2 \cdot \mathrm{R}_{3}, \quad \quad \mathcal{T}_{\mathcal{H}_{\ell_{m}^{(2)}}} \in\left\{3 \cdot \mathrm{R}_{1}+1 \cdot \mathrm{R}_{3}, 2 \cdot \mathrm{R}_{1}+2 \cdot \mathrm{R}_{2}\right\} .
$$


Therefore, it follows from relations $(\mathfrak{a})$ and $(\mathfrak{c})$ that $\mathcal{F}$ possesses two radial singularities $m_{1}, m_{2}$ of order 3 on the line $\ell_{m}^{(1)}$ and a radial singularity $m_{3}$ of order 2 or 3 on the line $\ell_{m}^{(2)}$.

We will see that the radiality order of the singularity $m_{3}$ of $\mathcal{F}$ is necessarily 3 , i.e. $\tau\left(\mathcal{F}, m_{3}\right)=4$. By $[2$, Proposition 2, page 23], the fact that $\tau\left(\mathcal{F}, m_{1}\right)+\tau\left(\mathcal{F}, m_{3}\right) \geq 4+3>\operatorname{deg} \mathcal{F}$ implies the invariance by $\mathcal{F}$ of the line $\ell=\left(m_{1} m_{3}\right)$; if $\tau\left(\mathcal{F}, m_{3}\right)$ were equal to 3 , then relations $(\mathfrak{a}),(\mathfrak{b})$ and $(\mathfrak{c})$, combined with the convexity of the foliation $\mathcal{H}_{\ell}$, would imply that $\mathcal{T}_{\mathcal{H}_{\ell}}=1 \cdot \mathrm{R}_{1}+1 \cdot \mathrm{R}_{2}+1 \cdot \mathrm{R}_{3}$ so that (see Table 1 ) $\mathcal{H}_{\ell}$ would possess a singularity $m^{\prime}$ on the line $\ell$ satisfying $\operatorname{CS}\left(\mathcal{H}_{\ell}, \ell, m^{\prime}\right) \in\left\{-\frac{13+2 \sqrt{13}}{13},-\frac{13-2 \sqrt{13}}{13}\right\}$ which is not possible.

By construction, the three points $m_{1}, m_{2}$ and $m_{3}$ are not aligned. We have thus shown that $\mathcal{F}$ admits three non-aligned radial singularities of order 3. By [3, Proposition 6.3] the foliation $\mathcal{F}$ is linearly conjugated to the FERMAT foliation $\mathcal{F}_{0}^{4}$.

Let us now consider the eventuality $\operatorname{CS}\left(\mathcal{H}_{\ell_{m}^{(1)}}, \ell_{m}^{(1)}, m\right)=\operatorname{CS}\left(\mathcal{H}_{\ell_{m}^{(2)}}, \ell_{m}^{(2)}, m\right)=-1$ for any choice of $m \in \Sigma$. In this case, Table 1 leads to $\mathcal{T}_{\mathcal{H}_{m}^{(i)}}=3 \cdot \mathrm{R}_{2}$ for $i=1,2$. Then, as before, by using relations $(\mathfrak{a}),(\mathfrak{b})$ and $(\mathfrak{c})$, we obtain that $\mathcal{F}$ possesses exactly three radial singularities of order 2 on each line $\ell_{m}^{(i)}$. Moreover, every line joining a radial singularity of order 2 of $\mathcal{F}$ on $\ell_{m}^{(1)}$ and a radial singularity of order 2 of $\mathcal{F}$ on $\ell_{m}^{(2)}$ must contain necessarily a third radial singularity of order 2 of $\mathcal{F}$. We can then choose a homogeneous coordinate system $[x: y: z] \in \mathbb{P}_{\mathbb{C}}^{2}$ so that the points $m_{1}=[0: 0: 1], m_{2}=[1: 0: 0]$ and $m_{3}=[0: 1: 0]$ are radial singularities of order 2 of $\mathcal{F}$. Moreover, in this coordinate system the lines $x=0, y=0, z=0$ must be invariant by $\mathcal{F}$ and there exist $x_{0}, y_{0}, z_{0} \in \mathbb{C}^{*}$ such that the points $m_{4}=\left[x_{0}: 0: 1\right], m_{5}=\left[1: y_{0}: 0\right], m_{6}=\left[0: 1: z_{0}\right]$ are radial singularities of order 2 of $\mathcal{F}$. The equalities $v\left(\mathcal{F}, m_{1}\right)=1, \tau\left(\mathcal{F}, m_{1}\right)=3$ and the invariance of the line $z=0$ by $\mathcal{F}$ ensure that every 1 -form $\omega$ defining $\mathcal{F}$ in the affine chart $z=1$ is of type

$$
\begin{aligned}
\omega= & (x \mathrm{~d} y-y \mathrm{~d} x)\left(\gamma+c_{0} x+c_{1} y\right)+\left(\alpha_{0} x^{3}+\alpha_{1} x^{2} y+\alpha_{2} x y^{2}+\alpha_{3} y^{3}\right) \mathrm{d} x+\left(\beta_{0} x^{3}+\beta_{1} x^{2} y+\beta_{2} x y^{2}+\beta_{3} y^{3}\right) \mathrm{d} y \\
& +\left(a_{0} x^{4}+a_{1} x^{3} y+a_{2} x^{2} y^{2}+a_{3} x y^{3}+a_{4} y^{4}\right) \mathrm{d} x+\left(b_{0} x^{4}+b_{1} x^{3} y+b_{2} x^{2} y^{2}+b_{3} x y^{3}+b_{4} y^{4}\right) \mathrm{d} y,
\end{aligned}
$$

where $a_{i}, b_{i}, c_{j}, \alpha_{k}, \beta_{k} \in \mathbb{C}$ and $\gamma \in \mathbb{C}^{*}$

In the affine chart $x=1$, resp. $y=1$, the foliation $\mathcal{F}$ is given by

$$
\begin{aligned}
\theta= & z^{3}\left(\gamma z+c_{0}+c_{1} y\right) \mathrm{d} y-\left(\alpha_{0} z+\alpha_{1} y z+\alpha_{2} y^{2} z+\alpha_{3} y^{3} z+a_{0}+a_{1} y+a_{2} y^{2}+a_{3} y^{3}+a_{4} y^{4}\right) \mathrm{d} z \\
& -\left(\beta_{0} z+\beta_{1} y z+\beta_{2} y^{2} z+\beta_{3} y^{3} z+b_{0}+b_{1} y+b_{2} y^{2}+b_{3} y^{3}+b_{4} y^{4}\right)(y \mathrm{~d} z-z \mathrm{~d} y), \\
\text { resp. } \eta= & -z^{3}\left(\gamma z+c_{0} x+c_{1}\right) \mathrm{d} x-\left(\beta_{0} x^{3} z+\beta_{1} x^{2} z+\beta_{2} x z+\beta_{3} z+b_{0} x^{4}+b_{1} x^{3}+b_{2} x^{2}+b_{3} x+b_{4}\right) \mathrm{d} z \\
& +\left(\alpha_{0} x^{3} z+\alpha_{1} x^{2} z+\alpha_{2} x z+\alpha_{3} z+a_{0} x^{4}+a_{1} x^{3}+a_{2} x^{2}+a_{3} x+a_{4}\right)(z \mathrm{~d} x-x \mathrm{~d} z) .
\end{aligned}
$$

A straightforward computation shows that

$$
\begin{array}{ll}
\left(J_{(y, z)=(0,0)}^{2} \theta\right) \wedge(y \mathrm{~d} z-z \mathrm{~d} y)=-z P(y, z) \mathrm{d} y \wedge \mathrm{d} z, & \left(J_{(x, z)=(0,0)}^{2} \eta\right) \wedge(z \mathrm{~d} x-x \mathrm{~d} z)=z Q(x, z) \mathrm{d} x \wedge \mathrm{d} z, \\
\left(J_{(x, y)=\left(x_{0}, 0\right)}^{2} \omega\right) \wedge\left(\left(x-x_{0}\right) \mathrm{d} y-y \mathrm{~d} x\right)=x_{0} R(x, y) \mathrm{d} x \wedge \mathrm{d} y, & \left(J_{(y, z)=\left(y_{0}, 0\right)}^{2} \theta\right) \wedge\left(\left(y-y_{0}\right) \mathrm{d} z-z \mathrm{~d} y\right)=-z S(y, z) \mathrm{d} y \wedge \mathrm{d} z, \\
\left(J_{(x, z)=\left(0, z_{0}\right)}^{2} \eta\right) \wedge\left(\left(z-z_{0}\right) \mathrm{d} x-x \mathrm{~d} z\right)=T(x, z) \mathrm{d} x \wedge \mathrm{d} z &
\end{array}
$$


with

$$
\begin{aligned}
P(y, z)= & a_{0}+a_{1} y+\alpha_{0} z+a_{2} y^{2}+\alpha_{1} y z, \\
Q(x, z)= & b_{4}+b_{3} x+\beta_{3} z+b_{2} x^{2}+\beta_{2} x z, \\
R(x, y)= & -x_{0}^{3}\left(\alpha_{0}+3 a_{0} x_{0}\right)+x_{0}^{2}\left(4 \alpha_{0}+11 a_{0} x_{0}\right) x+\left(\gamma+\alpha_{1} x_{0}^{2}+\beta_{0} x_{0}^{2}+2 a_{1} x_{0}^{3}+3 b_{0} x_{0}^{3}\right) y-2 x_{0}\left(3 \alpha_{0}+7 a_{0} x_{0}\right) x^{2} \\
& +\left(c_{0}-3 \alpha_{1} x_{0}-3 \beta_{0} x_{0}-5 a_{1} x_{0}^{2}-8 b_{0} x_{0}^{2}\right) x y+\left(c_{1}-\alpha_{2} x_{0}-\beta_{1} x_{0}-a_{2} x_{0}^{2}-2 b_{1} x_{0}^{2}\right) y^{2}+\left(3 \alpha_{0}+6 a_{0} x_{0}\right) x^{3} \\
& +\left(2 \alpha_{1}+3 \beta_{0}+3 a_{1} x_{0}+6 b_{0} x_{0}\right) x^{2} y+\left(\alpha_{2}+2 \beta_{1}+a_{2} x_{0}+3 b_{1} x_{0}\right) x y^{2}+\left(\beta_{2}+b_{2} x_{0}\right) y^{3}, \\
S(y, z)= & a_{0}+b_{0} y_{0}+a_{3} y_{0}^{3}+3 a_{4} y_{0}^{4}+b_{3} y_{0}^{4}+3 b_{4} y_{0}^{5}+\left(a_{1}+b_{1} y_{0}-3 a_{3} y_{0}^{2}-8 a_{4} y_{0}^{3}-3 b_{3} y_{0}^{3}-8 b_{4} y_{0}^{4}\right) y \\
& +\left(\alpha_{0}+\beta_{0} y_{0}-\alpha_{2} y_{0}^{2}-2 \alpha_{3} y_{0}^{3}-\beta_{2} y_{0}^{3}-2 \beta_{3} y_{0}^{4}\right) z+\left(a_{2}+3 a_{3} y_{0}+b_{2} y_{0}+6 a_{4} y_{0}^{2}+3 b_{3} y_{0}^{2}+6 b_{4} y_{0}^{3}\right) y^{2} \\
& +\left(\alpha_{1}+2 \alpha_{2} y_{0}+\beta_{1} y_{0}+3 \alpha_{3} y_{0}^{2}+2 \beta_{2} y_{0}^{2}+3 \beta_{3} y_{0}^{3}\right) y z, \\
T(x, z)= & -b_{4} z_{0}-z_{0}\left(a_{4}+b_{3}-c_{1} z_{0}^{2}-3 \gamma z_{0}^{3}\right) x+\left(b_{4}-\beta_{3} z_{0}\right) z-z_{0}\left(a_{3}+b_{2}+\beta_{1} z_{0}+2 c_{0} z_{0}^{2}\right) x^{2}+\left(\beta_{2}+3 c_{1} z_{0}+6 \gamma z_{0}^{2}\right) x z^{2} \\
& +\left(b_{3}-\alpha_{3} z_{0}-\beta_{2} z_{0}-3 c_{1} z_{0}^{2}-8 \gamma z_{0}^{3}\right) x z+\beta_{3} z^{2}-z_{0}\left(a_{2}+\alpha_{1} z_{0}\right) x^{3}+\left(b_{2}-\alpha_{2} z_{0}+\beta_{1} z_{0}+3 c_{0} z_{0}^{2}\right) x^{2} z,
\end{aligned}
$$

so that the equality $\tau\left(\mathcal{F}, m_{2}\right)=3\left(\right.$ resp. $\tau\left(\mathcal{F}, m_{3}\right)=3$, resp. $\tau\left(\mathcal{F}, m_{4}\right)=3$, resp. $\tau\left(\mathcal{F}, m_{5}\right)=3$, resp. $\tau\left(\mathcal{F}, m_{6}\right)=$ 3 ) implies that the polynomial $P$ (resp. $Q$, resp. $R$, resp. $S$, resp. $T$ ) is identically zero. From $P=Q=0$ we obtain $a_{0}=a_{1}=a_{2}=\alpha_{0}=\alpha_{1}=b_{4}=b_{3}=b_{2}=\beta_{3}=\beta_{2}=0$. Next, from the equalities $R=S=T=0$ we deduce that

$$
\begin{aligned}
& c_{0}=2 \gamma y_{0} z_{0}\left(x_{0} y_{0} z_{0}+1\right), \quad c_{1}=-2 \gamma z_{0}, \quad \alpha_{2}=2 \gamma y_{0} z_{0}^{2}\left(x_{0} y_{0} z_{0}+2\right), \quad \alpha_{3}=-2 \gamma z_{0}^{2}, \quad \beta_{0}=2 \gamma x_{0} y_{0}^{3} z_{0}^{3}, \\
& \beta_{1}=-2 \gamma y_{0} z_{0}^{2}\left(2 x_{0} y_{0} z_{0}+1\right), \quad a_{3}=-2 \gamma y_{0} z_{0}^{3}, \quad a_{4}=\gamma z_{0}^{3}, \quad b_{0}=-\gamma y_{0}^{3} z_{0}^{3}, \quad b_{1}=2 \gamma y_{0}^{2} z_{0}^{3},
\end{aligned}
$$$$
\left(x_{0} y_{0} z_{0}\right)^{2}+x_{0} y_{0} z_{0}+1=0 \text {. }
$$

Let us set $\rho=x_{0} y_{0} z_{0}$; then $\rho^{2}+\rho+1=0$ and $\omega$ is of type

$$
\begin{aligned}
\omega= & \gamma(x \mathrm{~d} y-y \mathrm{~d} x)\left(1+2 y_{0} z_{0}(\rho+1) x-2 z_{0} y\right)+2 \gamma z_{0}^{2} y^{2}\left(y_{0}(\rho+2) x-y\right) \mathrm{d} x+\gamma z_{0}^{3} y^{3}\left(y-2 y_{0} x\right) \mathrm{d} x \\
& +2 \gamma y_{0} z_{0}^{2} x^{2}\left(y_{0} \rho x-(2 \rho+1) y\right) \mathrm{d} y+\gamma y_{0}^{2} z_{0}^{3} x^{3}\left(2 y-y_{0} x\right) \mathrm{d} y .
\end{aligned}
$$

This 1-form is linearly conjugated to

$$
\omega_{H}^{4}=\left(2 x^{3}-y^{3}-1\right) y \mathrm{~d} x+\left(2 y^{3}-x^{3}-1\right) x \mathrm{~d} y .
$$

Indeed, the fact that $\rho$ satisfies $\rho^{2}+\rho+1=0$ implies that

$$
\omega_{H}^{4}=\frac{9 y_{0} z_{0}^{2}}{\gamma(\rho-1)} \varphi^{*} \omega, \quad \text { where } \varphi=\left(\frac{2 \rho+1-(\rho+2) x-(\rho+2) y}{3 y_{0} z_{0}}, \frac{(\rho-1) x-(2 \rho+1) y+\rho+2}{3 z_{0}}\right) .
$$

\section{References}

[1] P. Baum and R. Bott. Singularities of holomorphic foliations. J. Differential Geometry, 7:279-342, 1972.

[2] M. Brunella. Birational geometry of foliations. IMPA Monographs, 1. Springer, Cham, 2015. xiv+130 pp.

[3] S. Bedrouni and D. Marín. Tissus plats et feuilletages homogènes sur le plan projectif complexe. To appear in Bull. Soc. Math. France, 2017.

[4] S. Bedrouni. Feuilletages de degré trois du plan projectif complexe ayant une transformée de Legendre plate. PhD thesis, University of Sciences and Technology Houari Boumediene, 2017. Available on https://arxiv.org/abs/1712.03895. 
[5] C. Camacho and P. Sad. Invariant varieties through singularities of holomorphic vector fields. Ann. of Math. (2), 115(3):579-595, 1982.

[6] D. Cerveau, J. Déserti, D. Garba Belko, and R. Meziani. Géométrie classique de certains feuilletages de degré deux. Bull. Braz. Math. Soc. (N.S.), 41(2):161-198, 2010.

[7] K. Cordwell, S. Gilbertson, N. Nuechterlein, K. M. Pilgrim, and S. Pinella. On the classification of critically fixed rational maps. Conform. Geom. Dyn., 19:51-94,2015.

[8] C. Favre and J. V. Pereira. Webs invariant by rational maps on surfaces. Rend. Circ. Mat. Palermo (2), 64(3):403$431,2015$.

[9] D. Marín and J. V. Pereira. Rigid flat webs on the projective plane. Asian J. Math. 17(1):163-191, 2013.

[10] J. V. Pereira. Vector fields, invariant varieties and linear systems. Ann. Inst. Fourier (Grenoble), 51(5):1385-1405, 2001.

December 11, 2018

Samir Bedrouni, Faculté de Mathématiques, USTHB, BP 32, El-Alia, 16111 Bab-Ezzouar, Alger, Algérie E-mail: sbedrouni@usthb.dz

DAVID MARín, Departament de Matemàtiques Universitat Autònoma de Barcelona E-08193 Bellaterra (Barcelona) Spain E-mail : davidmp@mat.uab.es 